recognised by human HLA-DRB1*0401 restricted T cells in a 'citrulline'-specific fashion. These peptides can be recognised by $T$ cells from HLA-DR4+ ACPA+ RA patients, as shown in a first inventory.

\section{A187 IDENTIFICATION OF CITRULLINATED VIMENTIN PEPTIDES AS T CELL EPITOPES IN HLA-DR4 POSITIVE RA PATIENTS}

A L Feitsma, ${ }^{1}{ }^{2}$ E I H van der Voort, ${ }^{1}$ K L M C Franken, ${ }^{2}$ H Bannoudi, ${ }^{1}$ B G Elferink, ${ }^{2}$ J W Drijfhout, ${ }^{2}$ TW J Huizinga, ${ }^{1}$ R R P de Vries, ${ }^{2}$ REM Toes, ${ }^{1}$ A loan-Facsinay ${ }^{1}$ 'Department of Rheumatology, LUMC, Leiden, The Netherlands; ${ }^{2}$ Department of Immunohematology and Bloodtransfusion, LUMC, Leiden, The Netherlands

10.1136/ard.2010.129668x

Objective Antibodies directed against citrullinated proteins (ACPA) are highly specific for rheumatoid arthritis (RA). The production of ACPA is most likely dependent on the presence of $\mathrm{T}$ cells as ACPA have undergone isotype switching and associate with the shared epitope-containing HLA-DRB1 alleles (SE). Vimentin is a likely candidate protein for $\mathrm{T}$ cell recognition since over $90 \%$ of patients harbouring ACPA reactive with (peptides derived from) citrullinated vimentin carry SE-containing HLA-DRB1 alleles. The aim of this study was to identify citrullinated vimentin peptides presented to HLADRB1*0401 restricted T cells.

Methods HLA-DR4-transgenic mice were immunised with all possible citrulline-containing peptides derived from vimentin and $\mathrm{T}$ cell reactivity was analysed. Peptides recognised in a 'citrulline'-specific manner by $T$ cells were selected and analysed for their ability to be processed from the entire vimentin protein. A first inventory for recognition of selected epitopes by $\mathrm{T}$ cells from HLA-DR4+ ACPA+ RA patients was performed.

Results A 'citrulline'-specific response was observed for two of the peptides analysed. These peptides are naturally processed from the vimentin protein as citrullinated vimentin was recognised by peptide-specific $\mathrm{T}$ cells. T cell reactivity against these peptides was also observed in cell cultures from patients with RA.

Conclusion The authors have identified for the first time two naturally processed peptides from vimentin that are 